\title{
Endwall convective heat transfer for bluff bodies
}

\section{Wang, Lei; Salewski, Mirko; Sundén, Bengt; Borg, Andreas; Abrahamsson, Hans}

\section{Published in:}

International Communications in Heat and Mass Transfer

Link to article, DOI:

10.1016/j.icheatmasstransfer.2011.10.006

Publication date:

2012

Link back to DTU Orbit

Citation (APA):

Wang, L., Salewski, M., Sundén, B., Borg, A., \& Abrahamsson, H. (2012). Endwall convective heat transfer for bluff bodies. International Communications in Heat and Mass Transfer, 393(2), 167-173.

https://doi.org/10.1016/j.icheatmasstransfer.2011.10.006

\section{General rights}

Copyright and moral rights for the publications made accessible in the public portal are retained by the authors and/or other copyright owners and it is a condition of accessing publications that users recognise and abide by the legal requirements associated with these rights.

- Users may download and print one copy of any publication from the public portal for the purpose of private study or research.

- You may not further distribute the material or use it for any profit-making activity or commercial gain

- You may freely distribute the URL identifying the publication in the public portal

If you believe that this document breaches copyright please contact us providing details, and we will remove access to the work immediately and investigate your claim. 


\title{
Endwall convective heat transfer for bluff bodies
}

\author{
Lei Wang ${ }^{\mathrm{a}}$, Mirko Salewski ${ }^{\mathrm{b}}$, Bengt Sundén ${ }^{\mathrm{a}}$, Andreas Borg ${ }^{\mathrm{c}}$, Hans Abrahamsson ${ }^{\mathrm{c}}$ \\ ${ }^{a}$ Heat Transfer Division, Lund University, Box 118, SE-22100 Lund, Sweden \\ ${ }^{b}$ Ris $\phi$ National Laboratory for Sustainable Energy, Technical University of Denmark, DK-4000 Roskilde, Denmark \\ ${ }^{c}$ Volvo Aero Corporation, SE-46181 Trollhättan, Sweden
}

\begin{abstract}
The endwall heat transfer characteristics of forced flow past bluff bodies have been investigated using liquid crystal thermography (LCT). The bluff body is placed in a rectangular channel with both its ends attached to the endwalls. The Reynolds number varies from 50,000 to 100,000. In this study, a single bluff body and two bluff bodies arranged in tandem are considered. Due to the formation of horseshoe vortices, the heat transfer is enhanced appreciably for both cases. However, for the case of two bluff bodies in tandem, it is found that the presence of the second bluff body decreases the heat transfer as compared to the case of a single bluff body. In addition, the results show that the heat transfer exhibits Reynolds number similarity. For a single bluff body, the Nusselt number profiles collapse well when the data are scaled by $\mathrm{Re}^{0.55}$; for two bluff bodies arranged in tandem, the heat transfer scaling is changed to $\mathrm{Re}^{0.51}$, indicating that the power index of Reynolds number is flow dependent.
\end{abstract}

Keywords: endwall heat transfer, bluff bodies, heat transfer scaling, LCT

\section{Introduction}

In a gas turbine, endwall flows are the flows influenced by the inner hub and outer casing of the blades. These flows often contain horseshoe vortices that give rise to velocity components that are orthogonal to the primary flow direction (secondary flows). Basically, these vortices have a strong influence on the endwall heat transfer. In modern gas turbines, the endwall region becomes more and more thermally loaded. This requires that the endwall heat transfer be carefully considered in turbine designs, especially in the vicinity of the blade and downstream.

In laboratory experiments, the complicated blade is usually simplified as a circular cylinder or as a bluff body to gain insight into the physical mechanisms. Figure 1 shows a schematic picture of an incident flow approaching a circular cylinder [1]. Due to the strong adverse pressure gradient, the boundary layer undergoes a three-dimensional separation. The skewed boundary layer rolls up to form horseshoe vortices, resulting in two legs that wrap around the obstacle and trail downstream, as shown in Fig. 1. The size of the primary horseshoe vortex (V1) is of the same order-of-magnitude as the approaching boundary layer thickness; it is one order of magnitude larger than the secondary horseshoe vortex (V2). Moreover, V1 has the same sense of rotation as the approaching boundary layer vorticity, whereas V2 has the opposite sense of rotation to preserve the streamline topology. Because the primary horseshoe vortex entrains the higher-speed fluid from the free stream, the drag and the heat transfer are increased. The formation and development of the horseshoe vortices around bluff bodies have been studied exten-

Email address: lei.wang@energy.1th.se (Lei Wang) sively [2-5]. Some typical flow features were summarized in a review article by Simpson [6].

Concerning the heat transfer on the endwall, the fore junction of a bluff body is of particular interest. Praisner et al. [7] observed that the overall heat transfer rate in the fore junction region increased by as much as $200 \%$ compared to the unaffected regions due to the presence of the primary horseshoe vortex. Ireland and Jones [8] used the LCT technique to map the endwall heat transfer around the base plate of a bluff body. They reported that the Stanton number increased monotonically in the symmetry plane as the flow approached the obstacle. Goldstein et al. [9] investigated the mass transfer in the vicinity of a square bluff body by using a naphthalene sublimation technique. In contrast to [8], they noticed an obvious double-peak distribution of the Sherwood number upstream of the bluff body. The double peak was subsequently observed by Yoo et al. [10]. However, they also found that the second peak diminished as the angle of attack increased. More recently, Praisner and Smith [11] performed experiments that allowed simultaneous recording of the instantaneous flow velocity field and temporally-resolved heat transfer data on the endwall. They concluded that the second peak is associated with eruptive motions of the secondary horseshoe vortex.

The heat transfer downstream of an obstacle has also attracted attention. Fisher and Eibeck [12] studied the endwall heat transfer downstream of a streamlined cylinder and a circular cylinder by using a liquid crystal technique. The results indicated that the heat transfer is improved downstream of either obstacle. They attributed the enhanced heat transfer to the formation of horseshoe vortices. Ichimiya et al. [13] observed that the heat transfer pattern downstream of a cylindrical rod changes significantly as the Reynolds number varies from 1,000 


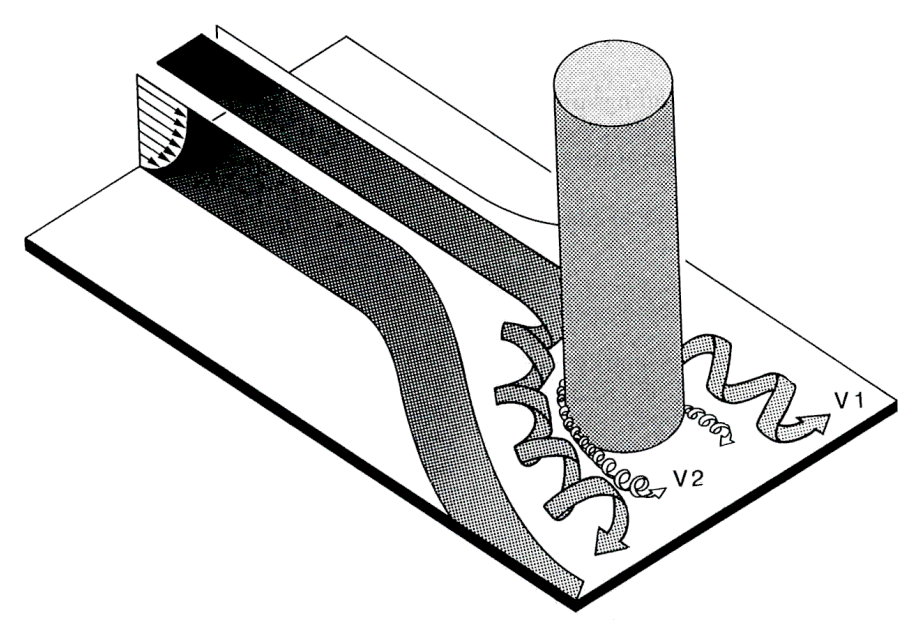

Figure 1: Schematic of horseshoe vortices, depicted by Goldstein and Karni [1].

to 10,000. In accordance with Fisher and Eibeck, they concluded that horseshoe vortices are responsible for the enhanced heat transfer. Wroblewski and Eibeck [14] examined the heat transfer mechanisms downstream of a tapered cylinder. By contrast, they suggested that the increased level of turbulence mixing is the main reason for the enhanced heat transfer.

In the present study, the endwall heat transfer of forced flow past bluff bodies is investigated. A single bluff body and two bluff bodies arranged in tandem are considered. The obstacle is placed such that both its ends are attached to the endwalls. The Reynolds number varies from 50,000 to 100,000. The heat transfer measurements are performed by using the LCT technique. The purposes of this paper are to fulfill three aspects, i.e., to report experimental observations, to provide detailed heat transfer data to validate numerical models, and to shed light on the cooling of gas turbines.

\section{Experimental setup and procedures}

The experiments were carried out in an open-circuit, suctiontype airflow channel. The channel is $5 \mathrm{~m}$ long. The width and height of the channel are $320 \mathrm{~mm}(W)$ and $80 \mathrm{~mm}(H)$, respectively. This results in a hydraulic diameter $D_{h}$ of $128 \mathrm{~mm}$. The channel is fabricated using $20-\mathrm{mm}$ thick transparent Plexiglas plates allowing for optical access for LCT measurements. A bell mouth is installed at the inlet of the facility to provide a uniform flow. The area ratio of the inlet contraction is 6:1. Air at ambient temperature from the laboratory room is drawn through the inlet contraction into the channel by a centrifugal fan situated at the downstream end. The maximum power of the fan is $3 \mathrm{~kW}$. By adjusting the frequency, the rotational speed of the fan can be regulated, and thus the mass flow rate can be controlled as desired. The whole channel consists of three sections, i.e., the inlet section ( $3.25 \mathrm{~m}$ long, which is equivalent to $25 D_{h}$ ), the test section (500 mm long), and the outlet section ( $1.25 \mathrm{~m}$ long). In the test section, heat transfer measurements are performed.

Figure 2 illustrates a schematic of the test section where the bluff body is fitted. The rectangular bluff body spans the height of the channel and is positioned on the centreline. The spanwise width $(b)$ of the bluff body is $80 \mathrm{~mm}$, which blocks the channel by $25 \%$ of its width, i.e., $b / W=25 \%$. The streamwise length $(l)$ of the bluff body is $40 \mathrm{~mm}$. The bluff body is made of Plexiglas due to the low thermal conductivity of the material $(0.2 \mathrm{~W} / \mathrm{m} \mathrm{K})$. For ease of inserting the obstacle, the test section is divided into two parts, and the top wall is removable. The bottom wall of the test section $(500 \mathrm{~mm} \times 320 \mathrm{~mm})$ is covered by a heating foil and is referred to as the heat transfer target wall. In order to minimize the heat conduction loss to the surrounding, a 50-mm thick Styrofoam sheet is used to cover the back of the bottom wall. The heat conductivity of Styrofoam is $0.03 \mathrm{~W} / \mathrm{m} \mathrm{K}$. The heater is made of an etched Inconel foil which is covered by a plastic film. Basically, a constant heat flux heating condition is established. The surface temperature is measured by an LCT sheet which is $500 \mathrm{~mm}$ long and $100 \mathrm{~mm}$ wide, as shown in Fig. 3. The LCT sheet (R35C5W, LCR Hallcrest Ltd) consists of a liquid crystal layer applied onto a Polyester (Mylar) substrate. Before the execution of the experiment, the LCT sheet was calibrated to get the relationship between the temperature and the hue value. The hue-temperature calibration data can be found in [15]. LCT images are captured by a GigE Vision CCD camera which allows $1600 \times 1200$ pixel resolution. The CCD camera is placed directly above the test section, as shown in Fig. 2. The spatial resolution of the LCT images is $0.22 \mathrm{~mm} /$ pixel. During the experiment, the CCD camera, illumination lighting, and the test section are covered by a dark enclosure to prevent the interference from the surrounding light noise.

A Cartesian coordinate system is defined in such a manner that the $\mathrm{x}$-axis is in the streamwise direction, the $\mathrm{y}$-axis is the wall-normal direction, and $\mathrm{z}$-axis is the spanwise direction. The origin is denoted by $\mathrm{O}$ and placed at the starting point of the test section, as shown in Fig. 2.

A liquid crystal technique enables one to evaluate the heat transfer pattern on the target wall. After steady state is achieved, a series of LCT images are generated, each corresponding to a distinct wall heat flux. In interpreting the temperature, the green colour is chosen as the reference colour 


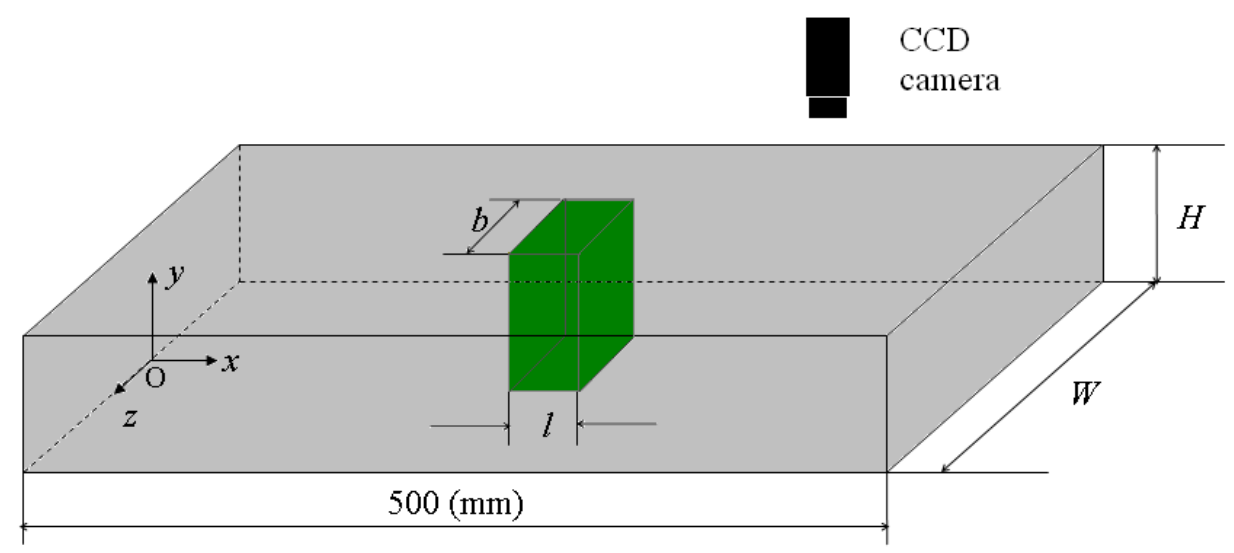

Figure 2: Specifications of the bluff body in the test section.

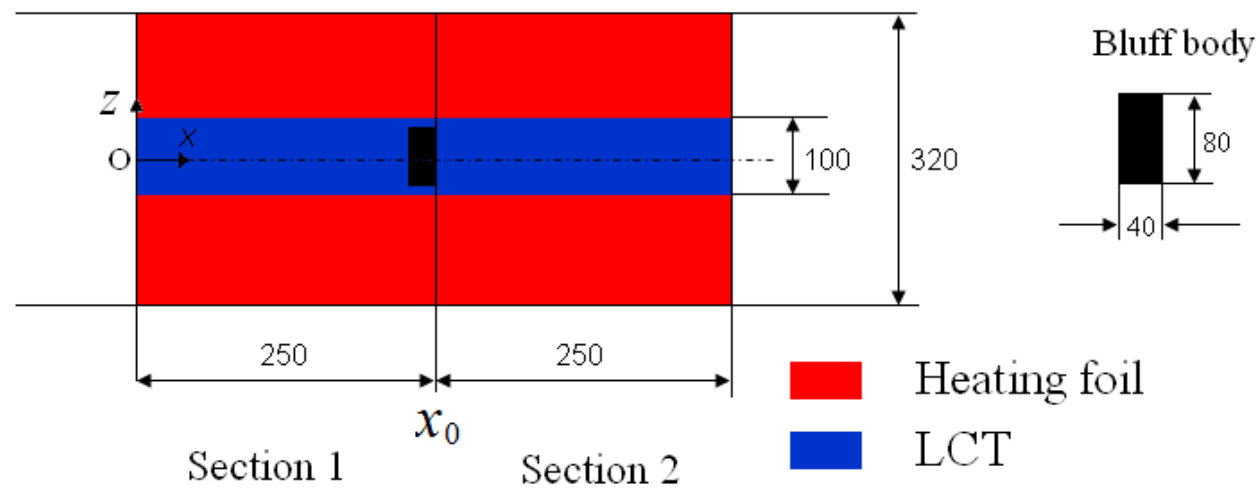

Figure 3: Configuration of the heat transfer target wall.

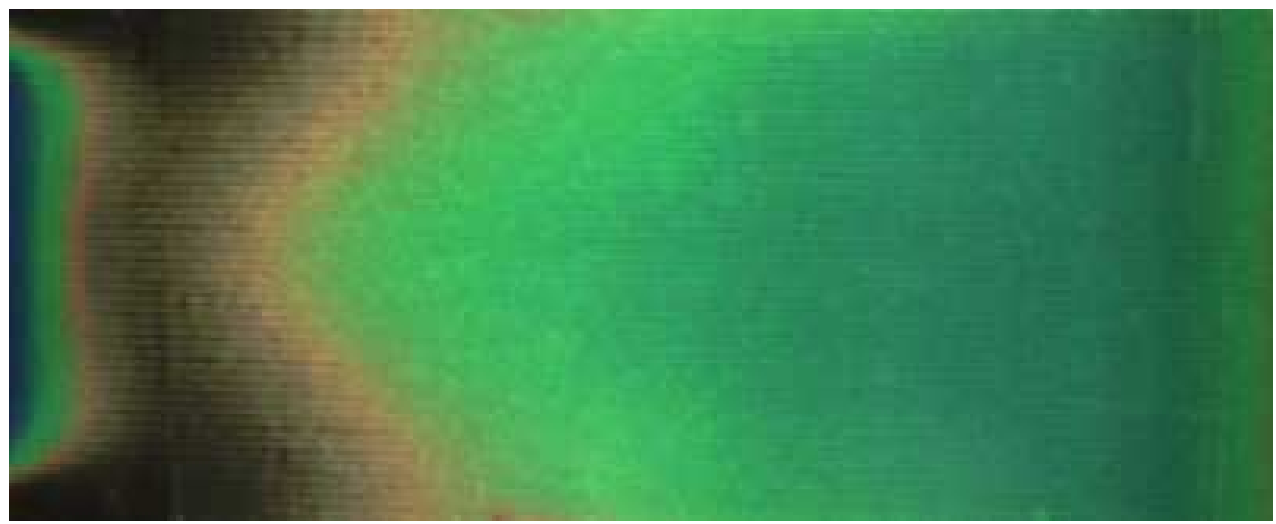

Figure 4: A typical LCT image behind a single bluff body where $\dot{q}_{w}=1850 \mathrm{~W} / \mathrm{m}^{2}$ and $\operatorname{Re}=100,000$. 
because it is the most temperature-sensitive colour in the calibration curve. The green colour is characterized by the hue value ranging from 60 to 100 which corresponds to a temperature variation between $35.5^{\circ} \mathrm{C}$ and $36.5^{\circ} \mathrm{C}$. By proper adjustment of the wall heat flux, the reference colour is moved from one location to another such that the entire area of interest is eventually covered with the reference colour. Thereby the heat transfer coefficient over the entire area can be obtained. In the present study, 10-20 LCT images are acquired to extract the local heat transfer coefficients depending on the test conditions. Figure 4 shows a typical LCT image behind a single bluff body, where the heat flux is $1850 \mathrm{~W} / \mathrm{m}^{2}$. The Reynolds number is 100,000. In Fig. 4, the green colour is used to calculate the wall temperature as well as the Nusselt number. For regions where the temperature is below the limit of the LCT (i.e., $35^{\circ} \mathrm{C}$ ), the LCT displays black colour.

\section{Data reduction}

The Reynolds number is here defined as

$$
\operatorname{Re}=U_{0} b / v
$$

where $U_{0}$ is the free-stream bulk velocity, and $b$ is the spanwise width of the bluff body. The free-stream bulk velocity $U_{0}$ was determined by measuring the maximum velocity at the axis of the channel. The maximum velocity $U_{\max }$ can be written as [16]

$$
U_{\max }=U_{0}+3.75 u^{*}
$$

where $u^{*}$ is the friction velocity obtained from the Moody chart at a given channel Reynolds number $\operatorname{Re}_{C}=U_{0} b / v$. In the present study, $\operatorname{Re}=50,000,70,000$, and 100,000 are considered.

For the forced convection, liquid crystal images are recorded in the steady state. When calculating the heat transfer coefficient, only points with temperature between $35.5^{\circ} \mathrm{C}$ and $36.5^{\circ} \mathrm{C}$ are processed; outside this range, no information is gained. The heat transfer coefficient $h$ is calculated based on Newton's law of cooling, i.e.,

$$
h=\dot{q}_{w} /\left(T_{w}-T_{i n}\right)
$$

where $\dot{q}_{w}$ is the wall heat flux, and $T_{w}$ and $T_{i n}$ are, respectively, the wall temperature and the inlet air temperature. When calculating the heat flux $\dot{q}_{w}$, the radiative and conductive heat losses to the surroundings are estimated. Using a diffuse gray-surface model, in which the emissivity is set to 0.8 , it is found that the radiative heat loss is less than $5 \%$ of the total heat supplied. The heat conduction loss across the wall is quite low (less than $1 \%$ of the total heat) and is negligible. In the present study, $\dot{q}_{w}$ varies from 700 to $2500 \mathrm{~W} / \mathrm{m}^{2}$ depending on the test conditions.

The heat transfer coefficient is represented in terms of Nusselt number $\mathrm{Nu}$, which is defined as

$$
\mathrm{Nu}=h b / k
$$

where $k$ is the thermal conductivity of air. Uncertainty analysis is performed by applying the method proposed by Moffat [17].
The uncertainty in the local heat transfer coefficients is estimated to be within $10 \%$ based on the $95 \%$ confidence level. This value takes into account the effects of the measuring errors in voltage, current, LCT reading, and the heat losses to the surroundings.

\section{Results and discussion}

\subsection{Test in the smooth channel flow}

Prior to the introduction of bluff bodies, the thermal development in the smooth channel flow was examined. As such, the Nusselt numbers in the first section $(0<x<250 \mathrm{~mm})$ were measured. Here, the hydraulic diameter of the channel is used as the characteristic length. At $\operatorname{Re}_{c}=160,000$, Fig. 5 presents the profile of $\mathrm{Nu} / \mathrm{Nu}_{0}$ which is plotted against the dimensionless streamwise distance $x / D_{h}$. The $\mathrm{Nu}_{0}$ is the Nusselt number for the fully developed channel flow and is calculated by the Dittus-Boelter correlation $\left(\mathrm{Nu}=0.023 \mathrm{Re}^{0.8} \operatorname{Pr}^{0.4}\right)$. It is observed that the Nusselt number drops gradually in the thermally developing region $0<x / D_{h}<1.2$; further downstream, the curve levels off and approaches a plateau with the value close to unity, indicating that the temperature field is fully developed. The discrepancy between the experimental data and the correlation is less than $10 \%$, which is within the range of measurement uncertainty. In what follows, with the presence of a bluff body in the channel, the heat transfer measurements are performed in the second section $(250 \mathrm{~mm}<x<500 \mathrm{~mm})$ where $x / D_{h}>2$.

\subsection{Heat transfer behind a bluff body}

In order to investigate the heat transfer characteristics behind a bluff body, the obstacle is placed at the end of the first section, as shown in Fig. 3. In this figure, the reference point is set at $x_{0}=250 \mathrm{~mm}$. Figure 6 demonstrates the contours of Nusselt number for $\mathrm{Re}=100,000$. The high heat transfer region $(\mathrm{Nu}>380)$ behind the bluff body manifests the footprints of horseshoe vortex legs that wrap around the bluff body. Because the vortex legs pump the low-momentum, warm fluid upward from the wall, the local heat transfer is enhanced. The lowest heat transfer is found in the region immediately behind the bluff body. In a wake region where $x>300 \mathrm{~mm}$, the heat transfer declines gradually due to the diminishing influence of the obstacle. Note that the Nusselt numbers at the end zone $(480 \mathrm{~mm}<x<500 \mathrm{~mm})$ are overestimated because the heat generation is lower at the edge of the heating foil. It should be pointed out that the heat transfer patterns for $\operatorname{Re}=50,000$ and 70,000 are similar to those illustrated in Fig. 6, thus, they are not shown here.

Further analysis of the heat transfer downstream of the bluff body is presented in Fig. 7, in which the Nusselt number profiles along the centreline (at $z=0$ ) are plotted. It is clear that all the Nusselt number profiles display a similar feature. There is a low heat transfer rate in the corner region immediately behind the bluff body, followed by a sharp rise which attains the maximum at $x=270 \mathrm{~mm}$. Further downstream, the Nusselt number drops gradually. Moreover, Fig. 7 shows that the heat transfer is a function of Reynolds number. An increase in Reynolds number generally gives rise to higher Nusselt number. 


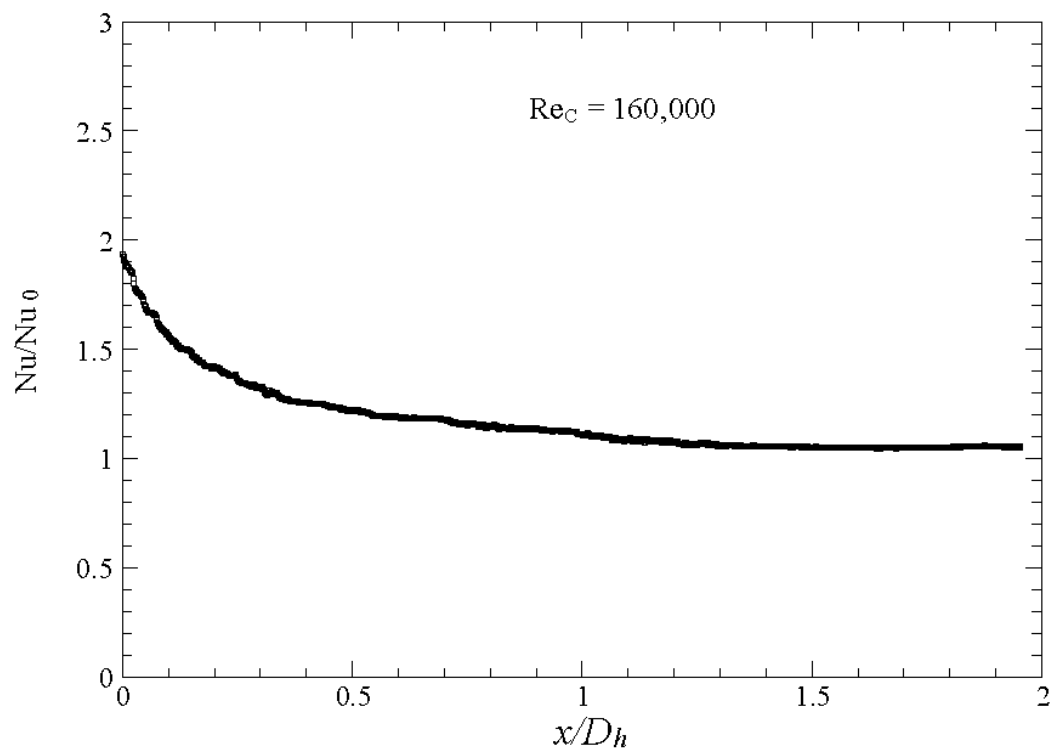

Figure 5: Thermal development region in the smooth channel flow.

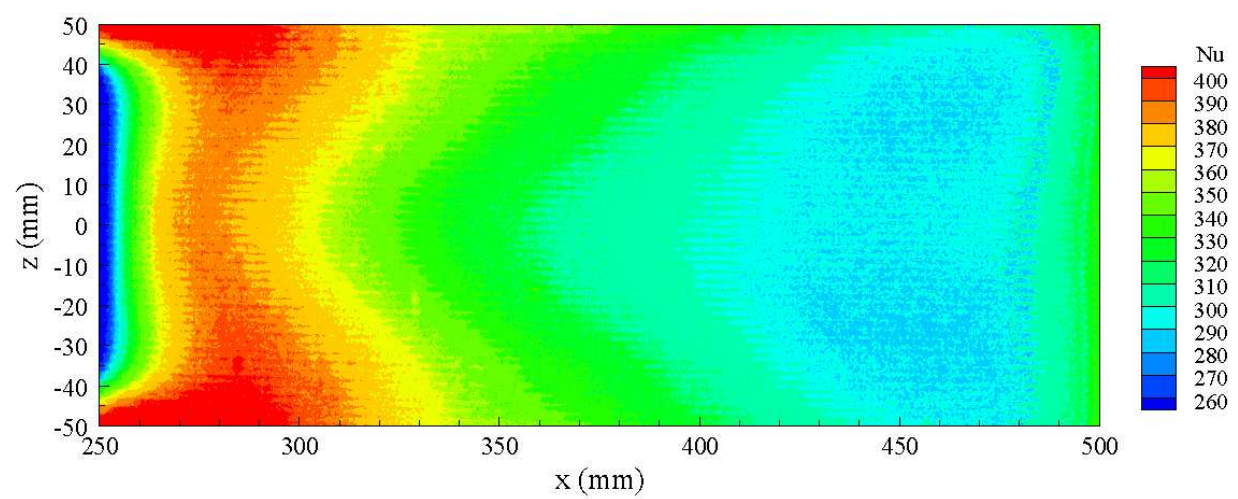

Figure 6: Nusselt number contours behind a single bluff body for $\operatorname{Re}=100,000$.

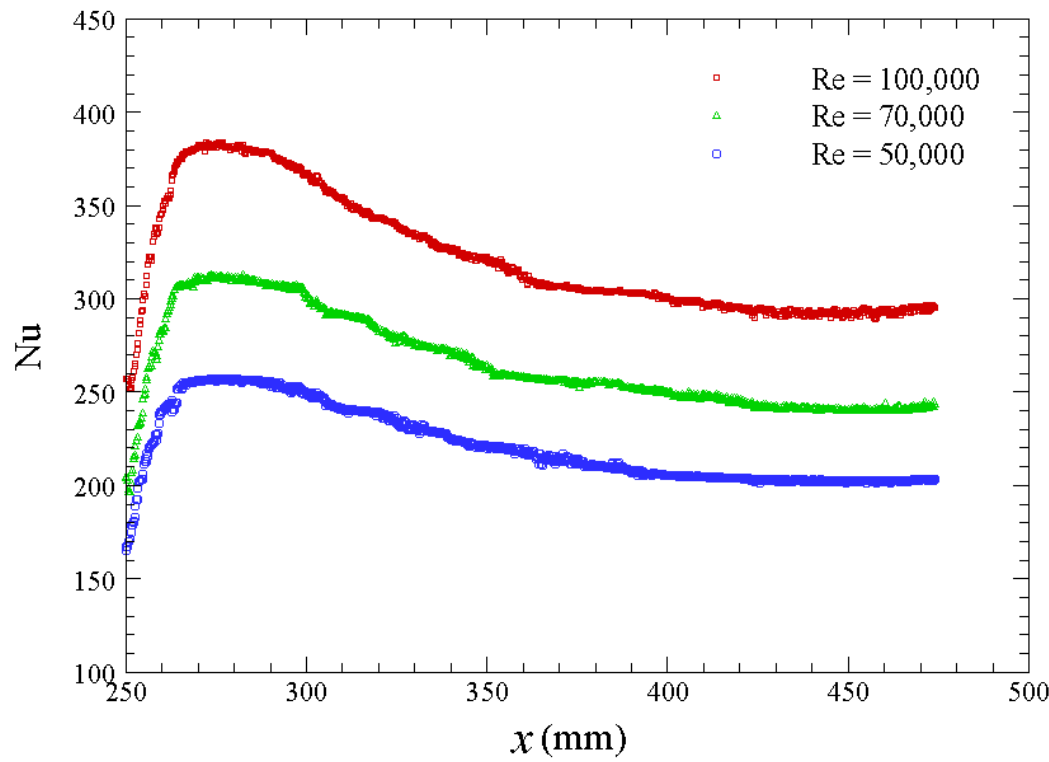

Figure 7: Nusselt number profiles along the centerline behind a single bluff body. 


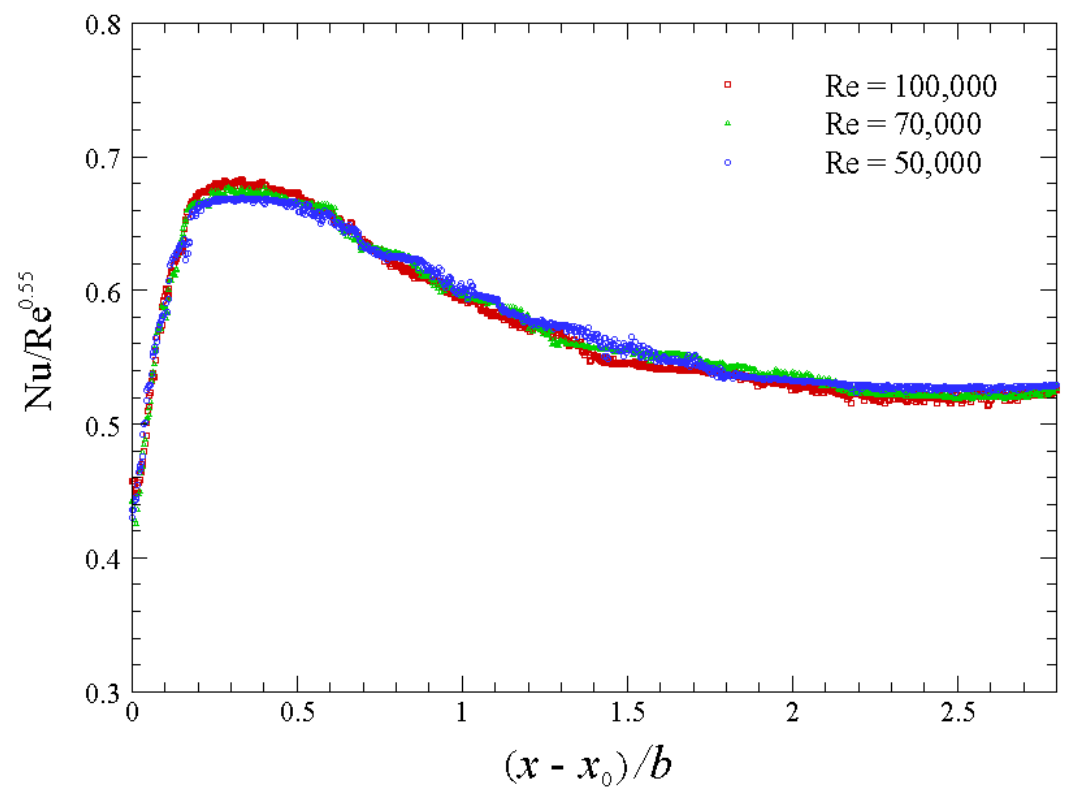

Figure 8: Scaled Nusselt number profiles behind a single bluff body. The data are scaled by $\operatorname{Re}^{0.55}$.

Since the shape of the Nusselt number profiles shown in Fig. 7 is similar for different Reynolds numbers, this triggers us to find a suitable scaling to make the profiles collapse. In many cases, the scaling for the Nusselt number has the form of $\mathrm{Re}^{n}$, where $n$ is a power index. For a given flow type, $n$ is approximately constant over a range of Reynolds numbers. In particular, if the normalized Nusselt number $\mathrm{Nu} / \mathrm{Re}^{n}$ is independent of the Reynolds number, i.e. $\mathrm{Nu} / \mathrm{Re}^{n}=f(x / L)$ where $L$ is a characteristic length, the heat transfer shows Reynolds number similarity. For example, Richardson [18] found that the flow over a circular cylinder which is held normal to a free stream has two distinct regions: a laminar boundary layer region $\left(\phi<80^{\circ}\right.$, where $\phi$ is the angle from the front stagnation point) and a separated flow region $\left(\phi>80^{\circ}\right)$. For the laminar flow, he found that the Nusselt numbers could be scaled by $\mathrm{Re}^{1 / 2}$ while for the turbulent separated flow, the scaling of $\mathrm{Re}^{2 / 3}$ was proposed. Nakamura and Igarashi [19] investigated unsteady heat transfer from a circular cylinder for Reynolds numbers from 3,000 to 18,900 . They found that the time-averaged Nusselt number profiles collapsed well onto a single curve in the laminar boundary layer flow region $\left(\phi<80^{\circ}\right)$ if the data were scaled by $\operatorname{Re}^{1 / 2}$. It should be pointed out that the power index $n$ may vary as the Reynolds number range is changed. For example, the $\mathrm{Re}^{2 / 3}$ scaling is only valid in the Reynolds number range of $10^{4}-10^{5}$. At lower Reynolds numbers, i.e., Re $1.5 \times 10^{4}$, the $2 / 3$ power law does not apply [19]. In the present study, the exponent $n$ is also sought to scale the heat transfer on the endwall. By performing a regression analysis, i.e., minimizing the sum of square residuals of the scaled Nusselt number curves, the optimum value 0.55 was found. Figure 8 illustrates the profiles of $\mathrm{Nu} / \mathrm{Re}^{0.55}$ along the centerline. The good agreement of scaled profiles clearly indicates that the heat transfer exhibits a similarity feature over the tested Reynolds number range. Note that the heat transfer in a turbulent channel flow is scaled by $\mathrm{Re}^{0.8}$. The lower power index of 0.55 for the endwall heat transfer scaling suggests that the flow structure downstream of a bluff body is quite different from that in the canonical turbulent channel flow.

\subsection{Heat transfer between two bluff bodies in tandem arrange- ment}

Figure 9 shows two in-line bluff bodies arranged in tandem. The spacing $(S)$ between the two obstacles is $250 \mathrm{~mm}$ and the ratio of $S / b$ is about 3 . Figure 10 presents the Nusselt number contours between two bluff bodies for $\mathrm{Re}=100,000$. A comparison with the Nusselt number contours behind a single bluff body (Fig. 6) shows that the heat transfer pattern in the wake region behind the first bluff body is not significantly altered by the presence of the second obstacle. Similar to Fig. 6, the footprints of the vortex legs that wrap around the bluff body are clearly revealed. However, further comparison of Fig. 10 with Fig. 6 shows that the Nusselt numbers in the region $(270 \mathrm{~mm}<x<420 \mathrm{~mm}$ ) behind the first bluff body are lower than those in the corresponding region behind a single bluff body. This may be explained by the fact that the flow is retarded as it encounters the adverse pressure gradient caused by the second bluff body. In the fore junction of the second bluff body $(420 \mathrm{~mm}<x<480 \mathrm{~mm})$, the heat transfer increases gradually as a result of the large-scale horseshoe vortex (V1). In the region just ahead of the bluff body ( $480 \mathrm{~mm}<x<500 \mathrm{~mm}$ ), a sharp rise in heat transfer is observed. Such highly elevated transfer coefficients near the upstream corner of the bluff body were also noted by Goldstein et al. [20]. This appears to be induced by the secondary vortex (V2) embedded in the front corner underneath the V1, as illustrated in Fig. 1. Incidentally, the Nusselt number contours for $\operatorname{Re}=50,000$ and 70,000 are not shown here because the heat transfer pattern is similar to that displayed in Fig. 10. 


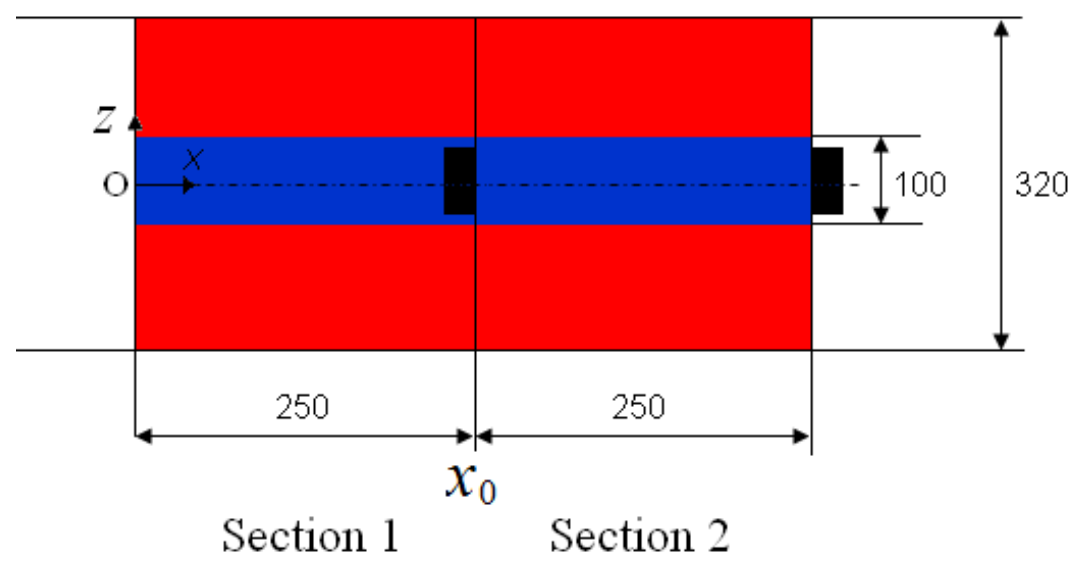

Figure 9: Two bluff bodies arranged in tandem.

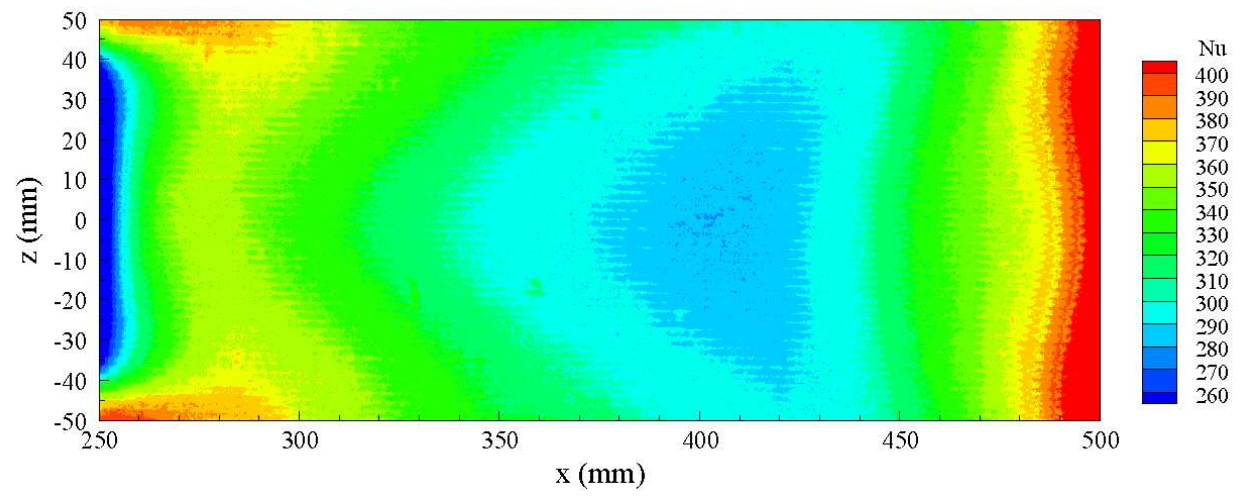

Figure 10: Nusselt number contours between two bluff bodies in tandem for $\mathrm{Re}=100,000$.

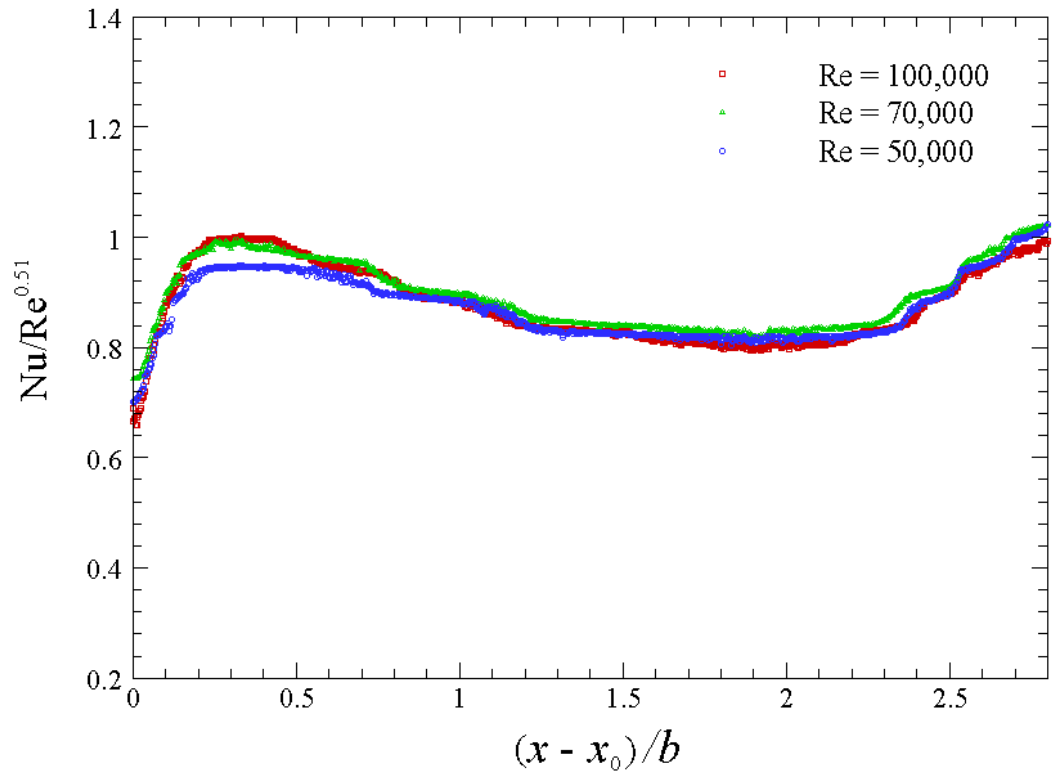

Figure 11: Scaled Nusselt number profiles between two bluff bodies in tandem. The data are scaled by $\mathrm{Re}^{0.51}$. 
Due to the interaction between the two bluff bodies in tandem arrangement, the heat transfer scaling may be altered. By performing a regression analysis, as stated in section 4.2 , it is found that the proper value of $n$ is 0.51 instead of 0.55 . Figure 11 shows the profiles of Nusselt number scaled by $\mathrm{Re}^{0.51}$ versus the dimensionless distance $\left(x-x_{0}\right) / b$, where $x_{0}=250 \mathrm{~mm}$. In general, the scaled profiles collapse onto a single curve for the tested Reynolds number range. This result suggests that the power index $n$ is flow dependent. Similar results were reported by Tanda [21]. He performed heat transfer measurements for a rectangular channel equipped with arrays of diamond-shaped elements. Both in-line and staggered arrays were considered. In the Reynolds number range 8,000 to 30,000, the results showed that the power index $n$ varied from 0.59 to 0.65 depending on the array configuration.

\section{Conclusions}

In the present study, liquid crystal measurements were carried out to investigate the endwall heat transfer of flow past bluff bodies. The bluff body is placed in a rectangular channel with both its ends attached to the endwalls. The Reynolds number varied from 50,000 to 100,000 . A single bluff body and two bluff bodies in tandem arrangement were considered. For both cases, the heat transfer is enhanced due to the formation of horseshoe vortices. However, for the case of two bluff bodies in tandem, the result shows that the heat transfer behind the first obstacle decreases as compared to that behind a single bluff body. Another interesting result is that the heat transfer exhibits Reynolds number similarity. For a single bluff body, it is found that the Nusselt numbers profiles collapse well onto a single curve when the data are scaled by $\mathrm{Re}^{0.55}$; for two bluff bodies arranged in tandem, the heat transfer scaling is changed to $\mathrm{Re}^{0.51}$, indicating that the power index of Reynolds number is flow dependent.

\section{Acknowledgments}

The current research is partly funded by the Swedish Research program TURBO POWER which is set up by the Swedish National Energy Agency (STEM), Volvo Aero Corporation and Siemens Industrial Turbomachinery. In addition, the Swedish Research Council (VR) provided additional financial support.

\section{References}

[1] R.J. Goldstein, and J. Karni, The effect of a wall boundary layer on local mass transfer from a cylinder in crossflow, ASME J. Heat Transfer, 106 (1984) 260-267.

[2] C.J. Baker, The laminar horseshoe vortex, J. Fluid Mech., 95 (1979) 347-367.

[3] C.J. Baker, The turbulent horseshoe vortex, J. Wind Eng. Ind. Aerodyn., 6 (1980) 9-23.

[4] C.J. Baker, The position of points of maximum and minimum shear stress upstream of cylinders mounted normal to flat plates, J. Wind Eng. Ind. Aerodyn., 18 (1985) 263-274.

[5] J.C.R. Hunt, C.J. Abell, J.A. Peterka, and H. Woo, Kinematical studies of the flow around free or surfacte-mounted obstacles; applying topology to flow visualization, J. Fluid Mech., 86 (1978) 179-200.

[6] R.L. Simpson, Junction flows, Ann. Rev. Fluid Mech., 33 (2001) 415-443.

[7] T.J. Praisner, C.V. Seal, L. Takmaz, and C.R. Smith, Spatialtemporal turbulent flow-field and heat transfer behavior in endwall junctions, Int. J. Heat Fluid Flow, 18 (1997) 142-151.

[8] P.T. Ireland, and T.V. Jones, Detailed measurements of heat transfer on and around a pedestal in fully developed passage flow, Proceedings 8th International Heat Transfer Conference, 3 (1986) 975-980.

[9] R.J. Goldstein, S.Y. Yoo, and M.K. Chung, Convective mass transfer from a square cylinder and its base plate, Int. J. Heat Mass Transfer, 33 (1990) 9-18.

[10] S.Y. Yoo, R.J. Goldstein, M.K. Chung, Effects of angle of attack on mass transfer from a square cylinder and its base plate, Int. J. Heat Mass Transfer, 36 (1993) 371-380.

[11] T.J. Praisner, and C.R. Smith, The dynamics of the horseshoe vortex and associated endwall heat transfer - Part 1: Temporal behavior, ASME J. Turbomach., 128 (2006) 747-754.

[12] E.M. Fisher, and P.A. Eibeck, The influence of a horseshoe vortex on local convective heat transfer, ASME J. Heat Transfer, 112 (1990) 329-335.

[13] K. Ichimiya, N. Akino, T. Kunugi, and K. Mitsushiro, Fundamental study of heat transfer and flow situation around a spacer (in the case of a cylindrical rod as a spacer), Int. J. Heat Mass Transfer, 31 (1988) 2215-2225.

[14] D.E. Wroblewski, and P.A. Eibeck, Turbulent heat transport in a boundary layer behind a junction of a streamlined cylinder and a wall, ASME J. Heat Transfer, 114 (1992) 840849.

[15] L. Wang and B. Sundén, Experimental investigation of local heat transfer in a square duct with various-shaped ribs, Heat Mass Transfer, 43 (2007) 759-766.

[16] H. Schlichting, Boundary-Layer Theory, Seventh Edition, 1979, McGraw Hill, New York.

[17] R.J. Moffat, Describing the uncertainties in experimental results, Exp. Thermal Fluid Sci., 1 (1998) 3-17.

[18] P.C. Richardson, Heat and mass transfer in turbulent separated flows, Chem. Eng. Sci., 18 (1963) 149-155.

[19] H. Nakamura, and T. Igarashi, Unsteady heat transfer from a circular cylinder for Reynolds numbers from 3000 to 15,000 , Int. J. Heat Fluid Flow, 25 (2004) 741-848.

[20] Goldstein, R. J., Yoo, S. Y., and Chung, M. K., Convective mass transfer from a square cylinder and its base plate, Int. J. Heat Mass Transfer, 22 (1990) 9-18.

[21] G. Tanda, Heat transfer and pressure drop in a rectangular channel with diamond-shaped elements, Int. J. Heat Mass Transfer, 44 (2001) 3529-3541. 